\title{
1 Can genome-based analysis be the new gold-standard 2 for routine Salmonella serotyping?
}

3 Sangeeta Banerji ${ }^{1 \S}$, Sandra Simon ${ }^{1 \S}$, Andreas Tille ${ }^{2}$ and Antje Flieger ${ }^{1 *}$

$4{ }^{1}$ Robert Koch-Institute, Division of Enteropathogenic Bacteria and Legionella (FG11) / National 5 Reference Centre for Salmonella and other Bacterial Enteric Pathogens, Burgstrasse 37, 38855

6 Wernigerode, Germany

$7{ }^{2}$ Robert Koch-Institute, Department for Infectious Disease Epidemiology (FG31), Burgstrasse 37,

838855 Wernigerode, Germany

$9{ }^{5}$ both authors contributed equally

$10 \quad{ }^{*}$ Corresponding author

11 Contact: fliegera@rki.de 


\section{Abstract}

Salmonella enterica is the second most reported bacterial cause of food-borne infections in Europe. Therefore molecular surveillance activities based on pathogen subtyping are an important measure of controlling Salmonellosis by public health agencies. In Germany, at the federal level, this work is carried out by the National Reference Center for Salmonella and other Bacterial Enteric Pathogens (NRC). With rise of next generation sequencing techniques, the NRC has introduced whole-genomebased typing methods for S. enterica in 2016. In this study we report on the feasibility of genomebased in silico serotyping in the German setting using raw sequence reads. We found that SeqSero and seven gene MLST showed $98 \%$ and $95 \%$ concordance, respectively, with classical serotyping for the here evaluated serotypes, including the most common German serotypes $S$. Enteritidis and $S$. Typhimurium as well as less frequently found serotypes. The level of concordance increased to $>99 \%$ when the results of both in silico methods were combined. However, both tools exhibited misidentification of monophasic variants, in particular monophasic S. Typhimurium and therefore need to be fine-tuned for reliable detection of this epidemiologically important variant. We conclude that with adjustments Salmonella genome-based serotyping might become the new gold standard. 
Subtyping of bacterial enteric pathogens, such as Salmonella enterica, traditionally relies on serotyping. The species Salmonella enterica is divided into six subspecies and consists of more than 2600 serovars, which are classified according to the White-Kauffmann- Le Minor Scheme ${ }^{1}$. Serotyping is based on determination of somatic $\mathrm{O}$ antigens and flagellin $\mathrm{H}$ antigens by reaction with specific antisera. Most $\mathrm{S}$. enterica serovars have two alternately expressed $\mathrm{H}$ antigens, also referred to as 'phases'. The phase- 1 and phase- 2 flagellin proteins are encoded by fliC and fljB, respectively. The phase switch is regulated by the invertase hin and the fliC repressor gene $\mathrm{flj}^{2}{ }^{2}$. Therefore, the specific antigenic formula consists of three positions: the first position represents the $O$ antigens, the second and third positions the two different flagellin $\mathrm{H}$ antigens. Each antigen position is separated by a colon, i.e. O:H1:H2. The antigenic formula for $\mathrm{S}$. Typhimurium for example is accordingly $1,4,[5], 12: i: 1,2$. There are variants of $S$. Typhimurium, which express only one flagellin and which therefore are referred to as monophasic $S$. Typhimurium. $S$. Enteritidis on the other hand does not possess a second flagellin per se, which is reflected in the antigenic formula: 1,9,12:g,m:-. It should be noted that some serovars share the same antigenic formula and require additional testing for unambiguous identification, e.g. the clinically important serovar $S$. Chloeraesuis shares its antigenic formula 6,7:c:1,5 with serovars $S$. Paratyphi $C$ and $S$. Typhisuis. A differentiation is possible based on biochemical characteristics or PCR ${ }^{3}$.

With rise of next generation sequencing (NGS) techniques, genomic typing tools have become increasingly popular and effective. Several in silico classification tools employing NGS data are available for Salmonella. The serotyping tools are either based on identifying and characterizing the serotype-determining genes or derive the serotype from in silico Multi Locus Sequence Typing (MLST) or a combination of both methods. MLST-based serotyping was sparked by the observation of Achtmann et al. that the phylogeny derived from MLST sequence types correlates with serotypes ${ }^{4,5}$. Achtmann and his group are also the developers of Enterobase, a platform for the phylogenetic analysis of selected bacteria, including Salmonella ${ }^{6}$. A report of the Establishing Next Generation Sequencing Ability for Genomic Analysis in Europe (ENGAGE) consortium identified four serotyping tools, specifically Metric-Oriented Sequence Typer (MOST), SeqSero, SalmonellaTypeFinder and SISTR, which were benchmarked for their performance and were found to have correlation rates between $65 \%$ and $88 \%$ with classical serotyping (http://www.engageeurope.eu/resources/benchmarking). MOST is a pipeline developed and employed by Public Health England, which infers an MLST type with a modified version of the program SRST, which was developed for deducing a sequence type from short reads, and utilizes a local database for identification of corresponding serotypes ${ }^{7,8}$. SeqSero is an in silico serotyping program and determines the presence of $\mathrm{O}$ and $\mathrm{H}$ antigen loci within the NGS data, which correspond to the antigens involved in classical serotyping ${ }^{9}$. SalmonellaTypeFinder is a pipeline developed by the Danish Technical University, which runs SeqSero and determines the MLST type using an in-house MLST calling tool, and then both results are used for determination of the serotype (https://bitbucket.org/genomicepidemiology/salmonellatypefinder/src/master/). Another typing platform is SISTR, which predicts the serotype by a combination of in silico hybridization and extended MLST, incorporated into a 'Microbial in Silico Typing' engine ${ }^{10}$.

Classification of Salmonella by serotyping is especially important for epidemiological investigations and is often routinely performed in its full scheme at National Reference Centers or Laboratories. It is also implemented at the German National Reference Center for Salmonella and other Bacterial 
Enteric Pathogens (NRC). The NRC receives around 3,000-4,000 Salmonella isolates per year from human infections for further characterization. The most common serotypes submitted are $S$. Enteritidis and $S$. Typhimurium, followed by other broad host range serotypes like $S$. Infantis and $S$. Derby ${ }^{11}$. Since 2016 the NRC has been shifting towards NGS-based analysis ${ }^{12}$.

Our aim for this study was to estimate whether NGS-based serotyping was feasible as a means of replacing traditional serotyping in our setting. The success rate of classical serotyping depends on many factors (e.g. access to high quality antisera, training of staff, and experience with rare serotypes) and was found to average worldwide at $82 \%$ and for European countries at $89 \%$ correct results in $2007^{13}$. Whereas the $\mathrm{O}$ antigens are determined within a few hours, characterization of the $\mathrm{H}$ phases may require up to 7 days. If the NRC replaced classical serotyping with a genome-based in silico typing method, this method should ideally match the high reported success rate of classical serotyping $^{13}$. Genome-based typing tools have performed well in several studies with maximum reported concordance levels of approximately $92 \%$ for SeqSero ${ }^{9}$ and approximately $94 \%$ for SISTR ${ }^{10,14}$. However, previous studies used assembled genomes for in silico typing. Only very recently, Ibrahim and Morin also reported results obtained with paired reads using the web-based application of SeqSero $1.0^{15}$. Genome assembly requires additional time and computing resources, which is a drawback for routine analysis of a large number of genomes.

Our goal for this study was therefore to directly use raw reads in order to save time and computing resources. Thus, our requirements for the tools were that the input data should need minimal preprocessing and should potentially fit into our existing analysis pipeline (Ridom SeqSphere ${ }^{+}{ }^{16}$. Since we need to process a large number of sequences, offline availability was also of major importance. SeqSero fulfilled all of these requirements (when used as a command line tool). The other above mentioned tools did not as they either use different allele detection algorithms for determination of MLST sequence types than Enterobase (MOST and SalmonellaTypeFinder) or require an assembled genome (SISTR). Therefore we decided to assess the performance of SeqSero and the Enterobase MLST scheme from Achtman et al. for serotype prediction ${ }^{4}$.

\section{Results}

The aim of this study was to assess two in silico serotype prediction tools, namely SeqSero and MLST via SeqSphere/Enterobase for their performance in routine Salmonella typing at the NRC. We chose 520 Salmonella isolates, mainly of human origin and predominantly from the years 2014-2018 as the data set for analysis. The selection comprised very frequently found serotypes as well as less frequent serotypes (Table 1). We investigated a total of 20 different serotypes and also looked at monophasic variants as well as rough phenotypes.

\section{Data quality is an important bottleneck}

Initially, we did not set a quality threshold for the raw read sequence files. In the course of the analysis we noticed that analysis with SeqSero 1.0 and/or Ridom SeqSphere failed if the file sizes of the raw sequence reads were lower than average $(<50,000 \mathrm{~KB})$. Since Zhang et al. only included data for analysis with SeqSero 1.0 with a minimal coverage of 10 -fold, we aimed for the same quality threshold ${ }^{9}$. Given an average genome size of approximately $4.8 \mathrm{Mb}$ for Salmonella enterica subsp. enterica, we calculated that a theoretical coverage of $\geq 10$-fold could only be achieved by a minimal 
read number of 100,000 each for paired end reads with a theoretical read length of 250: theoretical coverage $=$ total number of reads $x$ length of each read $[\mathrm{bp}] /$ genome size $[\mathrm{bp}]$. Sequencing was repeated for cases not meeting the minimal read number (Fig. S1).

SeqSero analysis correctly predicted the serovar in $98 \%$ of the isolates

SeqSero 1.0 predicted the serotype in $84 \%$ of analyzed strains in accordance to the classical serovar. In additional $14 \%$ of the cases the antigenic formula was shared by more than one serovar and SeqSero 1.0 predicted all eligible serotypes, e.g. Choleraesuis, Typhisuis or Paratyphi C for the antigenic formula 6,7:c:1,5, which we rated as ambiguous. These cases require additional testing as they would if determined by classical serotyping. Therefore an ambiguous prediction was counted as a correlating result in the overall summary. The total rate of correlation (correlation + ambiguous prediction) with our laboratory results was therefore $98 \%$ (Table 1). Five cases of prediction failure (1\%) occurred, all of which involved failed prediction of the 0-7 antigen. Additionally, five cases (1\%) of miscorrelation were found, which concerned monophasic strains of $S$. Typhimurium and $S$. Choleraesuis (Table 1).

Monophasic variants are only predicted correctly if they lack the flagellin genes

17 out of 19 monophasic $S$. Typhimurium strains were correctly predicted by SeqSero 1.0 using raw reads (Table 1 ). In two cases, SeqSero 1.0 predicted phenotypically monophasic $S$. Typhimurium as biphasic. In order to investigate this discrepancy we analyzed the respective whole genome sequences by de novo assembly. The isolate ERR2003330 lacked approximately 250 nucleotides in the central part of the $f j B$ gene as well as the whole hin gene (Fig. S2). Expression of the phase-2 flagellin gene $f l j B$ is co-regulated by the invertase gene hin and the fliC repressor gene $f l j A$. Apparently a transposase, tnpA, had integrated into this region. This explains why the second phase could not be detected by classical serotyping. Since SeqSero 1.0 only checks whether the fliC and fljB alleles are present, it would explain why the lack of the hin gene was not detected by the program and the partial deletion of the $f j B$ gene might have been too small to be detectable when using raw reads. We noted that SeqSero 1.0 correctly predicted the isolate to be monophasic when the analysis was performed with a5-assembled contigs. During preparation of this manuscript a new version of SeqSero called SeqSero 2.0 was available from github (https://github.com/denglab/SeqSero 2.0) and we rechecked the two non-correlating results with SeqSero 2.0 in the default k-mer-based mode. The program correctly classified isolate ERR2003330 as monophasic, probably due to the partial deletion in the $f l j B$ gene. However, when we used SeqSero 2.0 with a5 assembled contigs it classified the isolate wrongly as biphasic S. Typhimurium. The second isolate ERR2003327 had a transposon integrated into the $f l j B$ gene most probably rendering it non-functional. This isolate was identified to be biphasic with both SeqSero 1.0 and the k-mer-based approach of SeqSero 2.0 when using raw reads, because the fljB gene is fully present but interrupted. When using SeqSero 1.0 and SeqSero 2.0 with a5 assembled contigs isolate ERR2003327 was correctly predicted to be monophasic by both versions of the program.

Phenotypically monophasic variants of other serovars harboring fliC and $f j j B$ were also not recognized by SeqSero 1.0 or SeqSero 2.0, in particular three strains of $S$. Choleraesuis var. Kunzendorf not expressing phase-1 flagellum gene fliC (ERR3264001, ERR3264026, ERR3264035). This corroborates the fact that phenotypic traits are sometimes difficult to detect by in silico measures. A monophasic 
variant of S. Paratyphi B variant Java was recognized as monophasic $S$. Paratyphi B by SeqSero 1.0 and was additionally recognized to be $L(+)$-tartrate positive by SeqSero 2.0 .

Serovar prediction of rough strains is possible by means of SeqSero

Importantly, SeqSero 1.0 was able to predict a serotype for five out of six isolates with a rough phenotype, where classical serotyping was not successful (ERR3263893: S. Typhimurium, ERR3263889: S. Typhimurium monophasic, ERR3263894 [S. e. subspecies II]: 58:z6:z39, ERR3263880: S. Typhimurium monophasic, and ERR3263875: S. Typhimurium monophasic). We classified this as a correlation. For the rough strain ERR3264036 SeqSero 1.0 did not generate a full antigenic formula. PCR analysis according to Woods et al. 2008 targeting a 12.8-kb region specific to $S$. Choleraesuis yielded the serotype Choleraesuis ${ }^{3}$. In this case, SeqSero 1.0 was only able to predict a partial antigenic formula for Choleraesuis (-:c:1,5). SeqSero 2.0 was likewise not able to provide a complete antigenic formula for this particular strain. When we mapped the raw reads against the respective wzy allele (locus tag EL48_RS10980), we found the allele and the surrounding region (EL48_RS10955EL48_RS11010) missing (Fig. S3). We conclude that the rough phenotype of this particular isolate had a genetic basis.

\section{SeqSero does not reliably predict the 0-7 antigen}

We found five cases of prediction failure when using SeqSero 1.0 and all five cases involved failed prediction of the 0-7 antigen, which is part of the epidemiologically important serovars $S$. Choleraesuis and S. Infantis (ERR3264036, ERR3264076, ERR3264063, ERR3264067, and ERR3264066). Except for Isolate ERR3264036, the remaining four cases had an intact wzy allele but only few reads mapped to the 0-7 locus (Fig. S4 and S5). When we performed the analysis with SeqSero 2.0 the k-mer based approach yielded a complete antigenic formula which correlated with the laboratory phenotypes for all cases except for the rough strain ERR3264036, where the wzy allele is missing.

SeqSero is well suited for routine high-throughput analysis of raw reads with the exception of atypical monophasic strains

In summary, SeqSero 1.0 is an easy to use tool, which is available as free software from the website of the developers, or as an official Debian package from the Debian website. Currently an alpha test version of SeqSero 2.0 is available on Github with additional features, e.g. k-mer based approach and integrated identification of the taxonomic ID with SalmID in the allele based mode for subspecies identification of ambiguous serovars. When using SeqSero 1.0 with Illumina paired end raw reads we achieved a correlation rate of $98 \%$. The reasons for initial miscorrelation were mainly low data quality, which could be resolved by repeating the sequencing (Fig. S1). SeqSero 1.0 was able to predict a serotype for all rough isolates, except one. It correctly predicted monophasic variants if the flagellin genes fliC and/or fljB were missing. However, if the flagellin genes were only disrupted and/or other genes required for flagellar expression / phase transition were missing, SeqSero 1.0 and SeqSero 2.0 were not always able to reliably recognize monophasic variants. We conclude that with the exception of atypical monophasic variants of $S$. Typhimurium and other serovars and genetically rough strains (i.e. lack of $O$ antigen determining genes) SeqSero is able to correctly predict the vast majority of common serovars circulating in Germany. 
MLST predicted the serotype in 95\% of Salmonella isolates in concordance to the classical serovar found by serotyping (Table 1). Notably, all six rough isolates were assigned to a sequence type and a corresponding serotype. The prediction differed in 25 cases (5\%) from the phenotypic classification all of which involved second phase miscorrelation. Figure 1 shows an UPGMA (unweighted pair group method with arithmetic mean) Tree based on MLST and color coded according to the serovar obtained by slide agglutination. As expected, there is a clear correlation between serotype and one or more closely related STs for the majority of isolates (Fig. 1). S. Enteritidis for example is distributed into the two closely related STs: ST 11 and ST 183. The S. Typhi isolates of our collection spread across five different but closely related STs: ST 1, ST2, ST 3677, ST 2173 and ST 2209 (Fig. 1).

Sequence types do not consistently correlate with detection of flagellin antigens

206

207

208

209

210

211

212

213

214

215

It is notable that for the majority of isolates in Enterobase the antigenic formula is not provided by the user. Nevertheless, the majority of Enterobase strains belonging to ST 34, which had an antigenic formula provided, represented monophasic S. Typhimurium (203 out of 209 isolates as of May 2019). Therefore we assigned all ST 34 strains to monophasic Typhimurium. Enterobase strains belonging to ST 19 were a mix of monophasic and biphasic Typhimurium. We opted to classify all ST 19 isolates as biphasic Typhimurium although this would result in a high error rate. We preferred this to no classification at all. We obtained correlating results between MLST and classical serotyping for 17 out of 19 (89.5\%) of our monophasic S. Typhimurium strains. Only 32 out of 52 biphasic S. Typhimurium belonged to ST 19 (61.5\%) and were therefore also classified as biphasic with MLST. 20 out of 52 (38.5\%) phenotypically biphasic Typhimurium belonged to ST 34 and were therefore wrongly classified as monophasic by MLST. We also checked whether the classification of monophasic and biphasic $S$. Typhimurium would be improved by clustering according to core genome MLST. Figure 2 depicts a minimum spanning tree of only monophasic and biphasic $S$. Typhimurium isolates (including three rough isolates) based on the Enterobase core genome MLST scheme. The isolates cluster according to their ST rather than to their flagellin expression.

The $S$. Choleraesuis isolates of our collection, phenotypically lacking FliC were also not correctly classified by MLST typing. In Enterobase monophasic $S$. Choleraesuis var. Kunzendorf predominantly belonged to ST 66, whereas our isolates belonged to ST 145. Interestingly, MLST distinguished the monophasic S. Paratyphi B var. Java as such, since ST 42 mostly consists of monophasic var. Java entries in Enterobase.

MLST-based serotype prediction additionally provides phylogenetic context

The majority of our serotypes could each be assigned to a single eBG: e.g. S. Typhimurium to eBG 1 , S. Enteritidis to eBG 4, S. Typhi to eBG 13 and S. Choleraesuis to eBG 6 (Table 2). This is also reflected in the phylogenetic tree, where the different STs, which comprise the same serovar and belong to the same eBG are located in the same branch (Fig. 1). This indicates that German strains belonging to these serovars stem from a common ancestor ${ }^{4,17}$. One advantage of MLST serotype prediction compared to SeqSero was that there was no ambiguous serotype prediction. Different serovars with the same antigenic formula split into distinct eBurst groups (e.g. S. Choleraesuis eBG 6 and $S$. Paratyphi C eBG 20). MLST additionally provided important phylogenetic information, e.g. the $S$. Derby strains in our collection were of a polyphyletic nature as they split into three different eBGs (Table 2 and Fig. 1). In conclusion, MLST-based serotype prediction also proved to be very successful 
with the draw-back of not being able to distinguish between monophasic and biphasic $S$. Typhimurium as well as between $S$. Choleraesuis and monophasic $S$. Choleraesuis var. Kunzendorf.

After performing both analyses independently, we combined SeqSero 1.0 and MLST and used both results for predicting the serotype. In general, there was good agreement between the two methods. In case of disagreement, we evaluated the sequences individually. There were 24 cases of disagreement between SeqSero and MLST all of which concerned phase variation. Since our findings indicated that MLST was not suited for identification of phase variation and SeqSero generally performed better in this regard, we rated the SeqSero result as more adequate. There was disagreement between SeqSero and MLST regarding 20 S. Typhimurium isolates of ST 34, which were classified as monophasic by MLST and biphasic by SeqSero. Since biphasic ST 34 isolates cannot be correctly classified by MLST we chose the SeqSero prediction for these cases. The same applied for monophasic ST 19 S. Typhimurium isolates, which were also not correctly classified by MLST. The two isolates, which carried a transposase in fljB (ERR2003330 and ERR2003327), were correctly predicted as monophasic by MLST and here we opted for the MLST prediction because we had already analyzed these isolates by mapping. In the 5 cases of prediction failure by SeqSero, we chose the MLST prediction as the serovar. This way, the percentage of correlation was increased to $>99 \%$. In summary the combination of both independent methods enabled the identification of potential misclassifications where a closer analysis was necessary and thus reduced the rate of error.

\section{Discussion}

In this study we evaluated two genome-based in silico approaches and their combination for predicting Salmonella serotypes and their suitability for replacing classical serotyping. Table 3 summarizes the advantages and drawbacks of the three typing methods. We found that both tested prediction methods, the in silico serotyping approach by SeqSero 1.0 and the indirect serotype prediction with MLST yielded excellent correlation with our laboratory-based results analyzing 520 isolates from our strain collection (98\% SeqSero, 95\% MLST). Since our collection lacked a representative selection of strains of rare serotypes or higher subspecies we cannot rate the performance in this regard. Nonetheless it was representative of the most common human strains in Germany.

Our collection also included a novel serovar, derived from an outbreak related to sesame seeds ${ }^{18}$. Interestingly, the antigenic formula of this novel serovar was correctly identified by SeqSero demonstrating its effectiveness for classifying novel serovars. Our correlation rate of $98 \%$ using raw reads matches very well the correlation rate determined by the developers of SeqSero of $98.7 \%$ using $308 \mathrm{CDC}$ strains ${ }^{9}$. However, the correlation rate found by Zhang and colleagues dropped to $92.6 \%$ when using a higher number of Isolates, i.e 3306 isolates from GenomeTrakr. Likewise, a recent study of 1041 environmental Salmonella isolates including a wider variety than our study yielded a correlation of $86 \%$ to classical serotyping ${ }^{15}$. Recently, the developers of SeqSero presented a new version of the program named SeqSero 2.0 at the International Symposium on Salmonella and salmonellosis $2018^{19}$. SeqSero 2.0 can use SalmID in the assembly mode for subspecies identification 
of ambiguous serovars (www.github.com/hcdeenbakker/salmID). We did not test the assembly mode since it required the additional program SalmID, which we did not include in our assessment. We tested SeqSero 2.0 in its default k-mer based mode for reassessment of the ten cases where SeqSero failed. We found that with the default settings, SeqSero 2.0 also did not consistently detect monophasic variants of $S$. Typhimurium but showed improved performance in cases of high sequence variability.

Our results indicate that SeqSero does not reliably predict monophasic variants, in particular monophasic S. Typhimurium. Monophasic S. Typhimurium lacking fljB are correctly classified by SeqSero but atypical monophasic variants where $f l j B$ is present may be misclassified as biphasic. This is a potentially crucial limitation of the program as monophasic variants, especially of $S$. Typhimurium, are epidemiologically important and the latter comprise approximately $2 / 3$ of the $S$. Typhimurium received at the $\mathrm{NRC}^{20-24}$. We suggest including the detection of additional factors to the $f l j B$ allele, which determine integrity of the second phase flagellar antigen. Also the algorithm for phase determination when using raw reads should be refined so that disruptions in the $\mathrm{fliC} / \mathrm{fljB}$ genes can be detected in spite of the fact that the gene is fully present.

Regarding MLST, it was foreseeable by examining the strains in Enterobase that a clear classification between monophasic and biphasic S. Typhimurium based on ST would not be possible. Achtman et al. did not find a correlation between ST and monophasic $S$. Typhimurium when they analyzed a large and diverse collection ${ }^{4}$. On the other hand, it was reported that Italian and UK monophasic $S$. Typhimurium strains belonged to ST $34{ }^{20,21}$. Petrovska et al. showed that the current monophasic epidemic $S$. Typhimurium strains evolved from at least three independent events ${ }^{21}$. The monophasic strains of our collection predominantly belong to the current European ST 34 epidemic clone, therefore a good correlation for monophasic strains of ST 34 was obtained with MLST. On the other hand, biphasic strains of ST 34 were misclassified as monophasic. We therefore conclude that the classical MLST scheme alone is not able to clearly distinguish between monophasic and biphasic $S$. Typhimurium due to their polyphyletic nature. Our results further indicate that clustering by core genome MLST does also not improve classification according to flagellin expression. Since recent studies have found $S$. Typhimurium regions, which seem characteristic for certain monophasic variants it may be possible to develop an additional scheme based on the presence/absence of such specific genes to reliably identify monophasic variants of $S$. Typhimurium ${ }^{21,24}$.

We obtained the highest correlation to classical serotyping when we combined the predictions of SeqSero and MLST because the two methods use independent approaches for serotype determination and thereby complemented each other. Since SeqSero directly generates an antigenic formula, we rated its output as more adequate than the indirect determination by MLST. Nonetheless, with the additional information provided by MLST, it was possible to clarify all ambiguous predictions by SeqSero because the serovars, which shared the same antigenic formula, had different STs. Our results also indicate that MLST might even perform better in classifying rough strains than SeqSero. The combined prediction increased robustness because miscorrelating predictions of the two programs gave rise to more detailed analysis. Currently there are two tools available, which use the combined prediction of in silico serotyping and MLST. One is SalmonellaTypeFinder, which uses SeqSero and MLST and thus has the potential of performing well (https://bitbucket.org/genomicepidemiology/salmonellatypefinder/src/master/). We did not evaluate this tool in our study because it uses a different MLST calling algorithm than we routinely do and has not been published yet. The second tool is SISTR, which predicts the serotype with the help 
of in silico genoserotyping and validates the results with core genome MLST ${ }^{10}$. We did not evaluate SISTR because it requires assembled genomes. However, it performed very well in a previous report $^{14}$. A combination of genome-based serotyping and MLST is also advocated by other governmental agencies like Public Health England, who use MOST and Public Health Agency of Canada who use SISTR ${ }^{14}$.

\section{Conclusion}

SeqSero is an in silico serotyping tool generating an antigenic formula directly comparable to classical serotyping. MLST provides important phylogenetic information and is able to distinguish serovars with the same antigenic formula. The concomitant use of both tools seems best suited for in silico strain characterization to obtain the utmost information and a robust prediction. Nevertheless, some improvements are necessary to differentiate monophasic from biphasic strains. If the serotype is predicted by these two independent methods, a disagreement could indicate a potential problem requiring further investigation. Since we obtained a correlation rate of $>99 \%$ for SeqSero in combination with MLST, we conclude that the here investigated in silico typing tools could in combination outperform the current gold standard of phenotypic serotyping and could become the new gold standard.

\section{Methods}

\section{Short read sequencing}

Whole genome sequencing was performed at the NRC or at the Robert Koch-Institute's sequencing core facility on a MiSeq benchtop sequencer using Illumina's MiSeq Reagent Kit v3, yielding 2 × 300 bp paired end reads. Adapter-clipped reads were obtained from the sequencing unit and used in this study without additional processing unless stated otherwise. Sequencing was repeated for cases not meeting the minimal read number of 100,000 (Fig. S1). The fastq files of the paired-end sequence reads are available from the European Nucleotide Archive under the project numbers PRJEB30317 \& PRJEB16326. Project PRJEB16326 is part of EU COMPARE (https://www.compare-europe.eu/) and a subset of the German samples of that project have been included in this study.

\section{SeqSero 1.0/SeqSero 2.0}

The SeqSero 1.0 command line tool was downloaded from Github (https://github.com/denglab/SeqSero) and an official Debian package was created, which is available from https://blends.debian.org/med/tasks/bio. The installed program was then embedded into a script for batch analysis. Illumina MiSeq paired-end reads were directly used for serotype prediction. Apart from choosing the correct mode for the input data, i.e. single-end, paired-end, interleaved or assembled, the program offers no additional options. During drafting of this manuscript an alpha test version of SeqSero 2.0 became available from Github (https://github.com/denglab/SeqSero 2.0). We used SeqSero 2.0 with its default setting ( $k$-mer based mode) only to analyze isolates where SeqSero 1.0 did not produce a correlating result to classical serotyping.

Ridom SeqSphere settings and allele calling procedure 
For MLST analysis we used the 7 gene MLST scheme from Achtman et al. embedded in the Ridom SeqSphere ${ }^{+}$software (Ridom GmbH, Münster, Germany) ${ }^{4}$. Please note, that in spite of the fact that the scheme recommends de novo assembly of raw reads, we used mapping in order to save time and resources. Using the raw reads, the pipeline quality-trimmed and mapped the Illumina MiSeq reads against the reference genome S. Typhimurium LT2 (GenBank AE006468.2) using the build-in Burrows-Wheler Aligner in the default mode. This ideally yielded allele numbers for the seven housekeeping genes and the corresponding sequence type (ST). If Ridom SeqSphere ${ }^{+}$was not able to assign a ST there were generally two reasons: either low data quality ('Target QC procedure failure') or it was a potential new ST. For cases of low sequence quality sequencing was repeated (Fig. S1). For phylogenetic analysis of monophasic and biphasic $S$. Typhimurium isolates the Enterobase core genome MLST scheme was used in SeqSphere ${ }^{+}$.

The obtained MLST sequence types were entered into Enterobase to find corresponding serotypes from the database and if available the e-burst groups (eBGs). eBGs determination is based on an algorithm, which identifies the relationship of isolates with similar genotypes ${ }^{17}$. Enterobase periodically confers official eBG numbers to new eBGs.

377 If Ridom SeqSphere ${ }^{+}$reported a potential new ST we uploaded the NGS data of the respective isolates to Enterobase in order to obtain an official ST.

De novo assembly and mapping

380

For isolates with non-correlating results de novo assembly was performed using A5 or SPAdes ${ }^{25,26}$ Some isolates where further analyzed by mapping the raw reads against specific loci using the Geneious mapper or Bowtie2 in Geneious (www.geneious.com).

\section{Data Availability}

The raw sequence reads analyzed in this study are publicly available at the European Nucleotide Archive under the project accession numbers PRJEB30317 and PRJEB16326. PRJEB16326 is part of COMPARE and a subset of the German samples has been included in the current study. An overview of all strains and metadata is given in Table $\mathrm{S} 1$.

\section{References}

1 WHO Collaborating Centre for Reference and Research on Salmonella. Antigenic formulae of the Salmonella serovars. 9edn, (1997).

2 Barco, L. et al. Molecular Characterization of "Inconsistent" Variants of Salmonella Typhimurium Isolated in Italy. Foodborne Pathog Dis 11, 497-499, doi:10.1089/fpd.2013.1714 (2014).

3 Woods, D. F. et al. Rapid Multiplex PCR and Real-Time TaqMan PCR Assays for Detection of Salmonella enterica and the Highly Virulent Serovars Choleraesuis and Paratyphi C. J Clin Microbiol 46, 4018-4022, doi:10.1128/Jcm.01229-08 (2008).

4 Achtman, M. et al. Multilocus Sequence Typing as a Replacement for Serotyping in Salmonella enterica. Plos Pathog 8, doi:10.1371/journal.ppat.1002776 (2012). 
4015 Alikhan, N. F., Zhou, Z. M., Sergeant, M. J. \& Achtman, M. A genomic overview of the

402 population structure of Salmonella. Plos Genet 14, doi:10.1371/journal.pgen.1007261 (2018).

Zhou Z., Alikhan N.F., Mohamed K., the Agama Study Group, Achtman M. The user's guide to comparative genomics with EnteroBase. Three case studies: micro-clades within Salmonella enterica serovar Agama, ancient and modern populations of Yersinia pestis, and core genomic diversity of all Escherichia. https://doi.org/10.1101/613554 (2019).

7 Tewolde, R. et al. MOST : a modified MLST typing tool based on short read sequencing. Peerj 4, doi:10.7717/peerj.2308 (2016).

Inouye, M., Conway, T. C., Zobel, J. \& Holt, K. E. Short read sequence typing (SRST): multilocus sequence types from short reads. Bmc Genomics 13, doi:10.1186/1471-2164-13-338 (2012).

9 Zhang, S. K. et al. Salmonella Serotype Determination Utilizing High-Throughput Genome Sequencing Data. J Clin Microbiol 53, 1685-1692, doi:10.1128/Jcm.00323-15 (2015).

10 Yoshida, C. E. et al. The Salmonella In Silico Typing Resource (SISTR): An Open WebAccessible Tool for Rapidly Typing and Subtyping Draft Salmonella Genome Assemblies. Plos One 11, doi:10.1371/journal.pone.0147101 (2016).

11 Hauser, E. et al. Clonal Dissemination of Salmonella enterica Serovar Infantis in Germany. Foodborne Pathog Dis 9, 352-360, doi:10.1089/fpd.2011.1038 (2012).

12 Simon, S. et al. Evaluation of WGS based approaches for investigating a food-borne outbreak caused by Salmonella enterica serovar Derby in Germany. Food Microbiol 71, 46-54, doi:10.1016/j.fm.2017.08.017 (2018).

13 Hendriksen, R. S. et al. WHO Global Salm-Surv external quality assurance system for serotyping of Salmonella isolates from 2000 to 2007. J Clin Microbiol 47, 2729-2736, doi:10.1128/JCM.02437-08 (2009).

14 Yachison, C. A. et al. The Validation and Implications of Using Whole Genome Sequencing as a Replacement for Traditional Serotyping for a National Salmonella Reference Laboratory. Front Microbiol 8, doi:10.3389/fmicb.2017.01044 (2017).

15 Ibrahim, G. M. \& Morin, P. M. Salmonella Serotyping Using Whole Genome Sequencing. Front Microbio/ 9, 2993, doi:10.3389/fmicb.2018.02993 (2018).

16 Junemann, S. et al. Updating benchtop sequencing performance comparison. Nat Biotechnol 31, 294-296, doi:10.1038/nbt.2522 (2013).

17 Feil, E. J., Li, B. C., Aanensen, D. M., Hanage, W. P. \& Spratt, B. G. eBURST: Inferring patterns of evolutionary descent among clusters of related bacterial genotypes from multilocus sequence typing data. J Bacteriol 186, 1518-1530, doi:10.1128/Jb.186.5.1518-1530.2004 (2004).

18 Schielke, A.* Simon, $\mathrm{S}$ et al. European-wide salmonellosis outbreak with a novel serotype (11:z41:e,n,z15) attributable to sesame products, 2016-2017. Eurosurveillance (accepted for publication).

19 Colin, P. International Symposium on Salmonella and salmonellosis. Food Microbiol 71, 1, doi:10.1016/j.fm.2017.12.009 (2018).

20 Mastrorilli, E. et al. A Comparative Genomic Analysis Provides Novel Insights Into the Ecological Success of the Monophasic Salmonella Serovar 4,[5],12:i:-. Front Microbiol 9, doi:10.3389/fmicb.2018.00775 (2018).

21 Petrovska, L. et al. Microevolution of Monophasic Salmonella Typhimurium during Epidemic, United Kingdom, 2005-2010. Emerging infectious diseases 22, 617-624, doi:10.3201/eid2204.150531 (2016). 
22 Alt, K. et al. Outbreak of Uncommon O4 Non-Agglutinating Salmonella Typhimurium Linked to Minced Pork, Saxony-Anhalt, Germany, January to April 2013. Plos One 10, doi:10.1371/journal.pone.0128349 (2015).

23 Hauser, E. et al. Pork Contaminated with Salmonella enterica Serovar 4,[5],12:i:-, an Emerging Health Risk for Humans. Appl Environ Microb 76, 4601-4610, doi:10.1128/Aem.02991-09 (2010).

24 Palma, F. et al. Genome-wide identification of geographical segregated genetic markers in Salmonella enterica serovar Typhimurium variant 4,[5],12:i:-. Sci Rep-Uk 8, doi:10.1038/s41598-018-33266-5 (2018).

25 Tritt, A., Eisen, J. A., Facciotti, M. T. \& Darling, A. E. An Integrated Pipeline for de Novo

26 Bankevich, A. et al. SPAdes: A New Genome Assembly Algorithm and Its Applications to Single-Cell Sequencing. J Comput Biol 19, 455-477, doi:10.1089/cmb.2012.0021 (2012).

\section{Acknowledgements}

We thank Monique Duwe, Marita Wahnfried and Susanne Kulbe for excellent technical assistance. We thank the RKI NGS sequencing team in Berlin and Wernigerode. We are also thankful to all laboratories that sent strains to the NRC. The authors acknowledge funding by the EU COMPARE project within the Horizon 2020 (AF).

\section{Author contributions}

SB performed the in silico analysis and drafted the manuscript. SS supervised the serotyping and sequencing, advised in cgMLST analysis and provided input to the manuscript. AT wrote scripts for batch analysis and extraction of results. AF conceived and supervised the project and provided input to the manuscript.

\section{Competing interests}

The authors declare no competing interests.

\section{Figure Legends}

Fig.1 Unweighted Pair Group Method with Arithmetic mean (UPGMA) tree of all investigated isolates based on 7-gene MLST. The tree shows that serovars correlate to STs. Colors are based on ST.

Fig.2 Minimal Spanning tree of monophasic and biphasic Typhimurium isolates based on the Enterobase core genome MLST scheme and 7-gene MLST. The tree reveals that $S$. Typhimurium isolates cluster according to ST rather than expression of flagellin. Colors are based on phase and STs. 
Table 1. Overview of serotype prediction with SeqSero and MLST. Serotype was first determined by classical serotyping. Whole genome sequences were then analyzed with SeqSero or MLST.

482 Correlation means that the predicted serotype was the same as the classically determined serovar.

483 Ambiguous means that the correct serotype was listed among others. Prediction failure means that 484 no complete antigenic formula was derived. Miscorrelation means that a wrong antigenic formula 485 was derived. Overall (\%) is the same as Total (\%) except that Ambiguous (\%) is added to Correlation 486 (\%). Final results are shown, i.e. after resequencing if data quality was not met.

\begin{tabular}{|c|c|c|c|c|c|c|c|c|c|c|c|c|c|}
\hline \multirow[t]{2}{*}{ Serotype } & \multirow{2}{*}{$\begin{array}{l}\text { Sequen- } \\
\text { ced } \\
\text { Isolates }\end{array}$} & \multicolumn{3}{|c|}{ Correlation } & \multicolumn{3}{|c|}{ Ambiguous } & \multicolumn{3}{|c|}{ Prediction failure } & \multicolumn{3}{|c|}{ Miscorrelation } \\
\hline & & $\begin{array}{l}\text { Seq- } \\
\text { Sero }\end{array}$ & MLST & $\begin{array}{l}\text { Seq- } \\
\text { Sero } \\
+ \\
\text { MLST }\end{array}$ & $\begin{array}{l}\text { Seq- } \\
\text { Sero }\end{array}$ & MLST & $\begin{array}{l}\text { Seq- } \\
\text { Sero } \\
+ \\
\text { MLST }\end{array}$ & $\begin{array}{l}\text { Seq- } \\
\text { Sero }\end{array}$ & MLST & $\begin{array}{l}\text { Seq- } \\
\text { Sero } \\
+ \\
\text { MLST }\end{array}$ & $\begin{array}{l}\text { Seq- } \\
\text { Sero }\end{array}$ & MLST & $\begin{array}{l}\text { Seq- } \\
\text { Sero } \\
+ \\
\text { MLST }\end{array}$ \\
\hline Agona & 3 & 3 & 3 & 3 & 0 & 0 & 0 & 0 & 0 & 0 & 0 & 0 & 0 \\
\hline Choleraesuis & 33 & 0 & 33 & 33 & $\begin{array}{l}30 \text { or } \\
\text { Typhisuis } \\
\text { or } \\
\text { Paratyphi C }\end{array}$ & 0 & 0 & 3 & 0 & 0 & 0 & 0 & 0 \\
\hline $\begin{array}{l}\text { Choleraesuis } \\
\text { monophasic }\end{array}$ & 3 & 0 & 0 & 0 & 0 & 0 & 0 & 0 & 0 & 0 & 3 & 3 & 3 \\
\hline Derby & 55 & 55 & 55 & 55 & 0 & 0 & 0 & 0 & 0 & 0 & 0 & 0 & 0 \\
\hline $\begin{array}{l}\text { 11:z41:e,n,z15 } \\
\text { (novel serovar) }\end{array}$ & 10 & 10 & 10 & 10 & 0 & 0 & 0 & 0 & 0 & 0 & 0 & 0 & 0 \\
\hline Enteritidis & 115 & 115 & 115 & 115 & 0 & 0 & 0 & 0 & 0 & 0 & 0 & 0 & 0 \\
\hline Infantis & 50 & 49 & 50 & 50 & 0 & 0 & 0 & 1 & 0 & 0 & 0 & 0 & 0 \\
\hline Kentucky & 7 & 7 & 7 & 7 & 0 & 0 & 0 & 0 & 0 & 0 & 0 & 0 & 0 \\
\hline Kintambo & 3 & 0 & 3 & 3 & $\begin{array}{l}3 \text { or } \\
\text { Washington }\end{array}$ & 0 & 0 & 0 & 0 & 0 & 0 & 0 & 0 \\
\hline Kottbus & 12 & 0 & 12 & 12 & $\begin{array}{l}12 \text { or } \\
\text { Ferruch }\end{array}$ & 0 & 0 & 0 & 0 & 0 & 0 & 0 & 0 \\
\hline Mbandaka & 15 & 15 & 15 & 15 & 0 & 0 & 0 & 0 & 0 & 0 & 0 & 0 & 0 \\
\hline Mikawasima & 10 & 10 & 10 & 10 & 0 & 0 & 0 & 0 & 0 & 0 & 0 & 0 & 0 \\
\hline Muenchen & 25 & 0 & 25 & 25 & $\begin{array}{l}25 \text { or } \\
\text { Virginia }\end{array}$ & 0 & 0 & 0 & 0 & 0 & 0 & 0 & 0 \\
\hline Paratyphi B & 6 & 6 & 6 & 6 & 0 & 0 & 0 & 0 & 0 & 0 & 0 & 0 & 0 \\
\hline $\begin{array}{l}\text { Paratyphi B } \\
\text { monophasic }\end{array}$ & 1 & 1 & 1 & 1 & 0 & 0 & 0 & 0 & 0 & 0 & 0 & 0 & 0 \\
\hline Paratyphi C & 2 & 0 & 2 & 2 & $\begin{array}{l}2 \text { or } \\
\text { Cholerae- } \\
\text { suis or } \\
\text { Typhisuis }\end{array}$ & 0 & 0 & 0 & 0 & 0 & 0 & 0 & 0 \\
\hline Poano & 2 & 2 & 2 & 2 & 0 & 0 & 0 & 0 & 0 & 0 & 0 & 0 & 0 \\
\hline Strathcona & 2 & 2 & 2 & 2 & 0 & 0 & 0 & 0 & 0 & 0 & 0 & 0 & 0 \\
\hline Stourbridge & 14 & 14 & 14 & 14 & 0 & 0 & 0 & 0 & 0 & 0 & 0 & 0 & 0 \\
\hline Sundsvall & 1 & 0 & 1 & 1 & $\begin{array}{l}1 \text { or } \\
\text { Soahanina } \\
\text { or Sundvall }\end{array}$ & 0 & 0 & 0 & 0 & 0 & 0 & 0 & 0 \\
\hline Typhi & 74 & 74 & 74 & 74 & 0 & 0 & 0 & 0 & 0 & 0 & 0 & 0 & 0 \\
\hline $\begin{array}{l}\text { Typhimurium } \\
\text { biphasic }\end{array}$ & 52 & 52 & 32 & 52 & 0 & 0 & 0 & 0 & 0 & 0 & 0 & 20 & 0 \\
\hline $\begin{array}{l}\text { Typhimurium } \\
\text { monophasic }\end{array}$ & 19 & 17 & 17 & 19 & 0 & 0 & 0 & 0 & 0 & 0 & 2 & 2 & 0 \\
\hline $\begin{array}{l}\text { Serologically } \\
\text { rough }\end{array}$ & 6 & 5 & 6 & 6 & 0 & 0 & 0 & 1 & 0 & 0 & 0 & 0 & 0 \\
\hline Total number & 520 & 437 & 495 & 517 & 73 & 0 & 0 & 5 & 0 & 0 & 5 & 25 & 3 \\
\hline Total (\%) & 100.0 & 84.0 & 95.2 & 99.4 & 14.0 & 0 & 0 & 1.0 & 0 & 0 & 1.0 & 4.8 & 0.6 \\
\hline Overall (\%) & 100.0 & 98.0 & 95.2 & 99.4 & - & - & - & 1.0 & 0 & 0 & 1.0 & 4.8 & 0.6 \\
\hline
\end{tabular}


Table 2. Overview of Serovars with corresponding MLST sequence types and e-Burst groups

\begin{tabular}{|c|c|c|c|}
\hline Salmonella Serotype (Enterobase) & Sequence type & $\begin{array}{l}\text { e-Burst } \\
\text { Group }\end{array}$ & $\begin{array}{l}\text { Number of } \\
\text { Isolates }\end{array}$ \\
\hline Agona & 13 & 54 & 3 \\
\hline Choleraesuis & 139 & 6 & 1 \\
\hline Choleraesuis & 145 & 6 & 36 \\
\hline Derby & 39 & 57 & 6 \\
\hline Derby & 774 & 57 & 1 \\
\hline Derby & 40 & 57 & 5 \\
\hline Derby & 71 & 244 & 2 \\
\hline Derby & 682 & 264 & 41 \\
\hline Enteritidis & 11 & 4 & 110 \\
\hline Enteritidis & 183 & 4 & 5 \\
\hline 11:z41:e,n,z15 & 2914 & 472 & 10 \\
\hline Infantis & 32 & 31 & 49 \\
\hline Infantis & 2283 & 31 & 1 \\
\hline Kentucky & 198 & 56 & 7 \\
\hline Kintambo & 407 & 400 & 1 \\
\hline Kintambo & 2839 & ND & 1 \\
\hline Kintambo & 5841 & ND & 1 \\
\hline Kottbus & 212 & 64 & 11 \\
\hline Kottbus & 1669 & 63 & 1 \\
\hline Mikawasima & 1815 & 247 & 10 \\
\hline Mbandaka & 413 & 62 & 15 \\
\hline Muenchen & 82 & 8 & 25 \\
\hline Paratyphi B & 86 & 5 & 6 \\
\hline Paratyphi B mono (var Java) & 42 & 32 & 1 \\
\hline Paratyphi C & 146 & 20 & 2 \\
\hline Poano & 557 & 87 & 2 \\
\hline Strathcona & 2559 & ND & 2 \\
\hline Stourbridge & 736 & 438 & 8 \\
\hline Stourbridge (only RKI data) & 3736 & 464 & 6 \\
\hline Sundsvall (first typed as Poano) & 488 & 305.2 & 1 \\
\hline Subsp. II & 781 & 340 & 1 \\
\hline Typhi & 1 & 13 & 38 \\
\hline Typhi & 2 & 13 & 32 \\
\hline Typhi & 2173 & 13 & 1 \\
\hline Typhi & 2209 & 13 & 1 \\
\hline Typhi & 3677 & 13 & 2 \\
\hline Typhimurium \& monophasic var. & 19 & 1 & 36 \\
\hline Typhimurium \& monophasic var. & 34 & 1 & 39 \\
\hline Total & 39 & $>26$ & 520 \\
\hline
\end{tabular}


bioRxiv preprint doi: https://doi.org/10.1101/806208; this version posted October 18, 2019. The copyright holder for this preprint (which was not certified by peer review) is the author/funder, who has granted bioRxiv a license to display the preprint in perpetuity. It is made available under aCC-BY-NC-ND 4.0 International license.

Table 3. Overview of advantages and drawbacks of the investigated typing methods and their sources of errors. Concerning classical serotyping we also referred to Hendriksen et al. $2009^{13}$.

\begin{tabular}{|c|c|c|c|c|}
\hline $\begin{array}{l}\text { Typing } \\
\text { Method }\end{array}$ & Advantage & Drawback & $\begin{array}{l}\text { Main reasons for } \\
\text { Errors }\end{array}$ & $\begin{array}{l}\text { How to address } \\
\text { sources of errors }\end{array}$ \\
\hline \multirow[t]{2}{*}{ Serotyping } & $\begin{array}{l}\text { Directly determines } \\
\text { phenotype }\end{array}$ & $\begin{array}{l}\text { No typing of rough } \\
\text { strains possible }\end{array}$ & $\begin{array}{l}\text { Lack of experience } \\
\text { with serotyping }\end{array}$ & $\begin{array}{l}\text { Intensively } \\
\text { trained staff }\end{array}$ \\
\hline & $\begin{array}{l}\text { Well established } \\
\text { method }\end{array}$ & $\begin{array}{l}\text { Requires high quality } \\
\text { antisera }\end{array}$ & & $\begin{array}{l}\text { Quality control } \\
\text { mechanism }\end{array}$ \\
\hline \multirow[t]{2}{*}{ SeqSero } & $\begin{array}{l}\text { Classification } \\
\text { analogous to } \\
\text { classical serotyping }\end{array}$ & $\begin{array}{l}\text { Genotype may not } \\
\text { correspond to } \\
\text { phenotype due to } \\
\text { undetected } \\
\text { mutations }\end{array}$ & $\begin{array}{l}\text { Low sequence data } \\
\text { quality }\end{array}$ & $\begin{array}{l}\text { Quality control } \\
\text { mechanism, e.g. } \\
\text { of sequencing } \\
\text { process }\end{array}$ \\
\hline & $\begin{array}{l}\text { No assembly } \\
\text { required } \\
\text { Can be automated }\end{array}$ & $\begin{array}{l}\text { High quality } \\
\text { sequencing data } \\
\text { required (e.g. } \\
\text { coverage, } \\
\text { contamination) }\end{array}$ & $\begin{array}{l}\text { Monophasic variants } \\
\text { are only determined } \\
\text { by lack of } f l j B\end{array}$ & $\begin{array}{l}\text { Improve } \\
\text { detection method } \\
\text { for monophasic } \\
\text { variants }\end{array}$ \\
\hline \multirow[t]{2}{*}{$\begin{array}{l}\text { MLST- } \\
\text { based } \\
\text { typing }\end{array}$} & $\begin{array}{l}\text { Provides } \\
\text { phylogenetic } \\
\text { information }\end{array}$ & $\begin{array}{l}\text { High quality } \\
\text { sequencing data } \\
\text { required (e.g. } \\
\text { coverage, } \\
\text { contamination) }\end{array}$ & $\begin{array}{l}\text { Low sequence data } \\
\text { quality }\end{array}$ & $\begin{array}{l}\text { Quality control } \\
\text { mechanism, e.g. } \\
\text { of sequencing } \\
\text { process }\end{array}$ \\
\hline & Can be automated & $\begin{array}{l}\text { Assembly } \\
\text { recommended }\end{array}$ & & \\
\hline
\end{tabular}




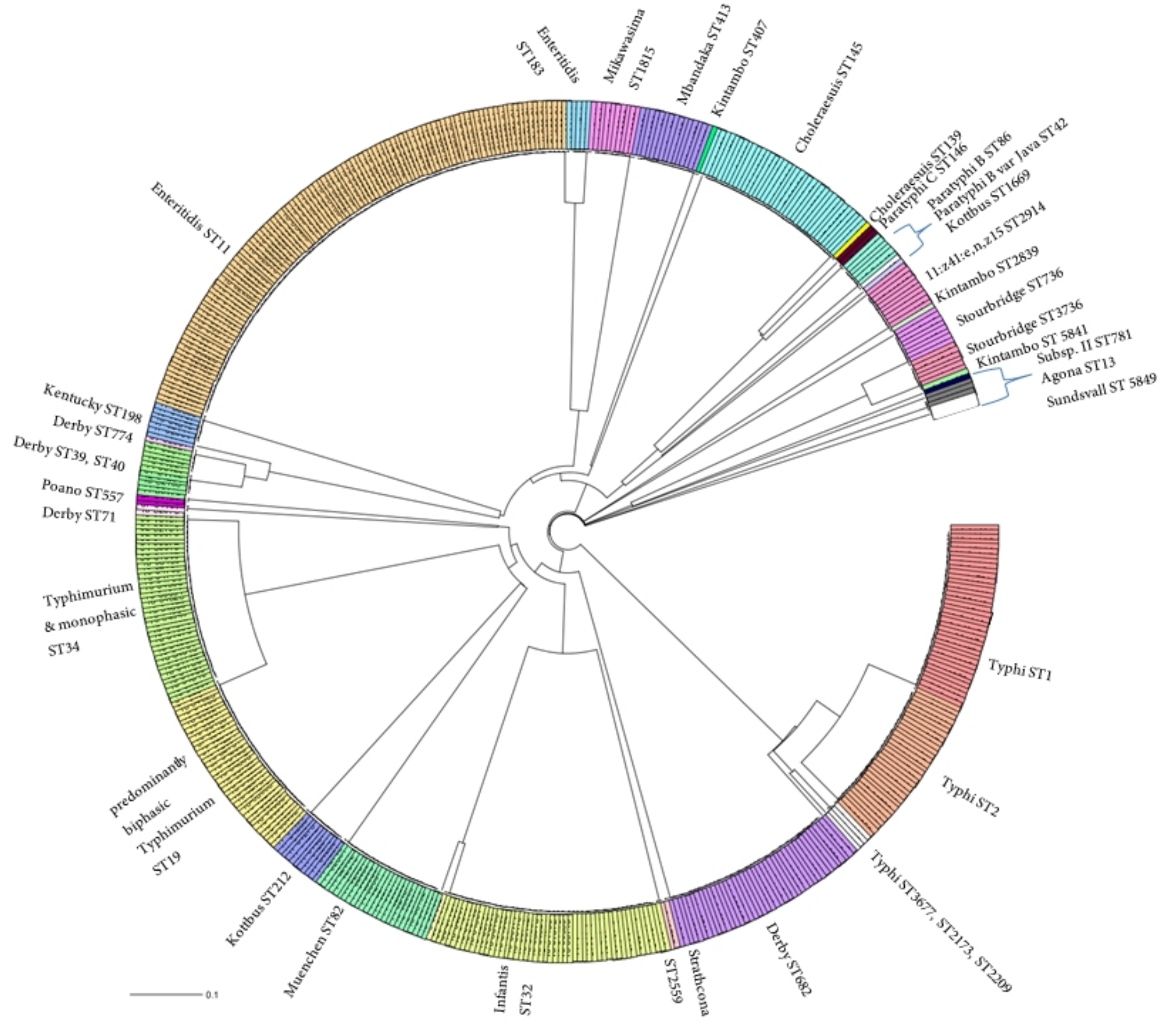


Typhimuirum mono ST34

Typhimurium biphasic ST34

Typhmurium biphasic ST19

Typhimurium mono ST19

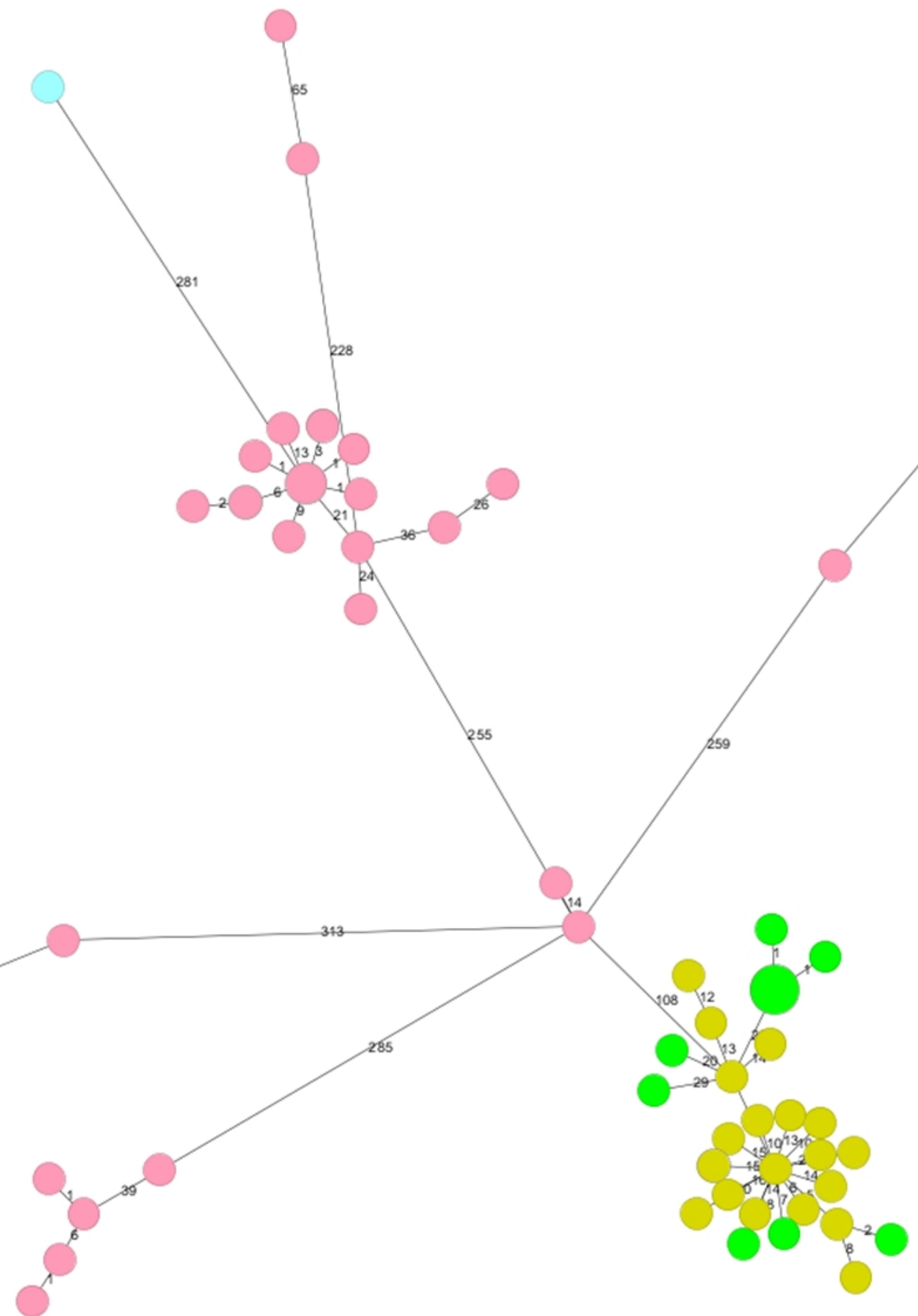

